\title{
On-Line Free Form Surface Measurement Via a Fuzzy-Logic Controlled Scanning Probe
}

\author{
Ming Chang \\ Chung Yuan Christian University \\ Paul P. Lin \\ Cleveland State University, p.lin@csuohio.edu
}

Follow this and additional works at: https://engagedscholarship.csuohio.edu/enme_facpub

Part of the Controls and Control Theory Commons, and the Manufacturing Commons How does access to this work benefit you? Let us know!

\section{Publisher's Statement}

NOTICE: this is the author's version of a work that was accepted for publication in International Journal of Machine Tools \& Manufacture. Changes resulting from the publishing process, such as peer review, editing, corrections, structural formatting, and other quality control mechanisms may not be reflected in this document. Changes may have been made to this work since it was submitted for publication. A definitive version was subsequently published in International Journal of Machine Tools \& Manufacture, 39, 4, (04-01-1999); 10.1016/S0890-6955(98)00052-2

\section{Original Citation}

Chang, M., , \& Lin, P. P. (1999). On-line free form surface measurement via a fuzzy-logic controlled scanning probe. International Journal of Machine Tools and Manufacture, 39(4), 537-552.

This Article is brought to you for free and open access by the Mechanical Engineering Department at EngagedScholarship@CSU. It has been accepted for inclusion in Mechanical Engineering Faculty Publications by an authorized administrator of EngagedScholarship@CSU. For more information, please contact library.es@csuohio.edu. 


\title{
On-line free form surface measurement via a fuzzy-logic controlled scanning probe
}

\author{
Ming Changa, Paul P. Lin ${ }^{\mathrm{b}, *}$ \\ "Mechanical Engineering Department, Chung Yuan Christian University, Chung Li, Taiwan \\ ${ }^{\mathrm{b}}$ Mechanical Engineering Department, Cleveland State University, Cleveland, OH 44115, USA
}

\section{Introduction}

In non-contact measurements, laser scanning through structured lighting [1], Moire method [2], and phase shift interferometry $[3,4]$ techniques have been developed and used in academia and industry. Although they provide high measuring accuracy, they are generally limited to some

* Corresponding author. 
conditions such as sensitivity to vibration and the inability to react to drastic change in surface curvature. In contrast, contact measurements are more reliable, but more time consuming. Traditionally, a touch trigger type of probe is installed on a CMM (coordinate measuring machine) for the purpose of 3-D geometry inspection [5]. However, this contact measurement technique suffers a major drawback for not being able to adjust the probe's orientation in order to keep the probe normal to the measured surface. Recently, scanning contact probes have been introduced and used in conjunction with a CMM. The contact probe used in this work is called SP2 (scanning probe 2) as the second in a series of high performance measuring probes, manufactured by Renishaw, UK, designed to be used to digitize or copy components in 3-D. The probe's low weight and high stiffness offers high measuring accuracy at high speeds.

In this work, the probe is installed on a CNC machine, in lieu of CMM, so that not only online surface measurement can be achieved, but also a component can be accurately made or reproduced. The probe has three linear axes giving true linear and parallel motion to the stylus. The linear motion ensures that the travel is consistently straight in each axis to within a few microns (micrometers), but it does not follow a curved path as with parallel spring motion probes or pivot motion probes. The parallel motion ensures the stylus remains at the same angle during its travel so that the flank of the stylus can be used for measurement. In addition, damping is incorporated into each axis of the probe to smooth out probe motion and to prevent oscillation when the probe stylus comes free from the part. One drawback is its inability to prevent unwanted lateral shift or movement when the probe is much in contact with the measured surface. Another drawback which is very common in continuous contact measurements is the inability to sense the change in surface curvature, which often results in interrupting the measuring process. Traditionally, a CNC machine is controlled through the most popular linear control scheme, the PID (Proportional-Integral-Derivative) control. However, due to unknown surface curvature, a required transfer function for the controlled plant cannot be specified in advance. Fuzzy logic control, on the other hand, is non-linear in nature and requires solely the sensing data. A fuzzy logic controller, in this case, can quickly take actions in response to the surface curvature change.

In this paper, the measuring system is first described and followed by the employment of fuzzy logic control, and free form surface determination. Finally, some experimental data are discussed and the conclusions are drawn.

\section{The measuring system}

The measuring system includes a CNC machine, a SP2 scanning probe, 3-axis simultaneous motion drivers, 6-axis 24 bits $\mathrm{AB}$ phase modulator and counter, and a personal computer. Fig. 1 shows the schematic diagram of the measuring system. In this system, the measured object is placed on top of a $X Y$ stage, the scanning probe is mounted on a CNC machine in $Z$ direction (vertically downward), the measuring signal goes to a digital signal processor and counter, and the $\mathrm{CNC}$ machine is driven in $X, Y$ and $Z$ axes simultaneously.

The scanning probe has maximum measurement range of $\pm 4.7 \mathrm{~mm}$ in $X, Y$ and $Z$ axes. The resolution in each axis is $2 \mu \mathrm{m}$ in each axis without further interpolation. The linearity and straightness are $\pm 2 \mu \mathrm{m}$ (typical) over $2 \mathrm{~mm}$ range and $\pm 4 \mu \mathrm{m}$ (typical) over $2 \mathrm{~mm}$ range in $X$, $Y$ and $Z$ axes, respectively. The probe's settling time is generally about $0.2 \mathrm{~s}$, but it may vary 


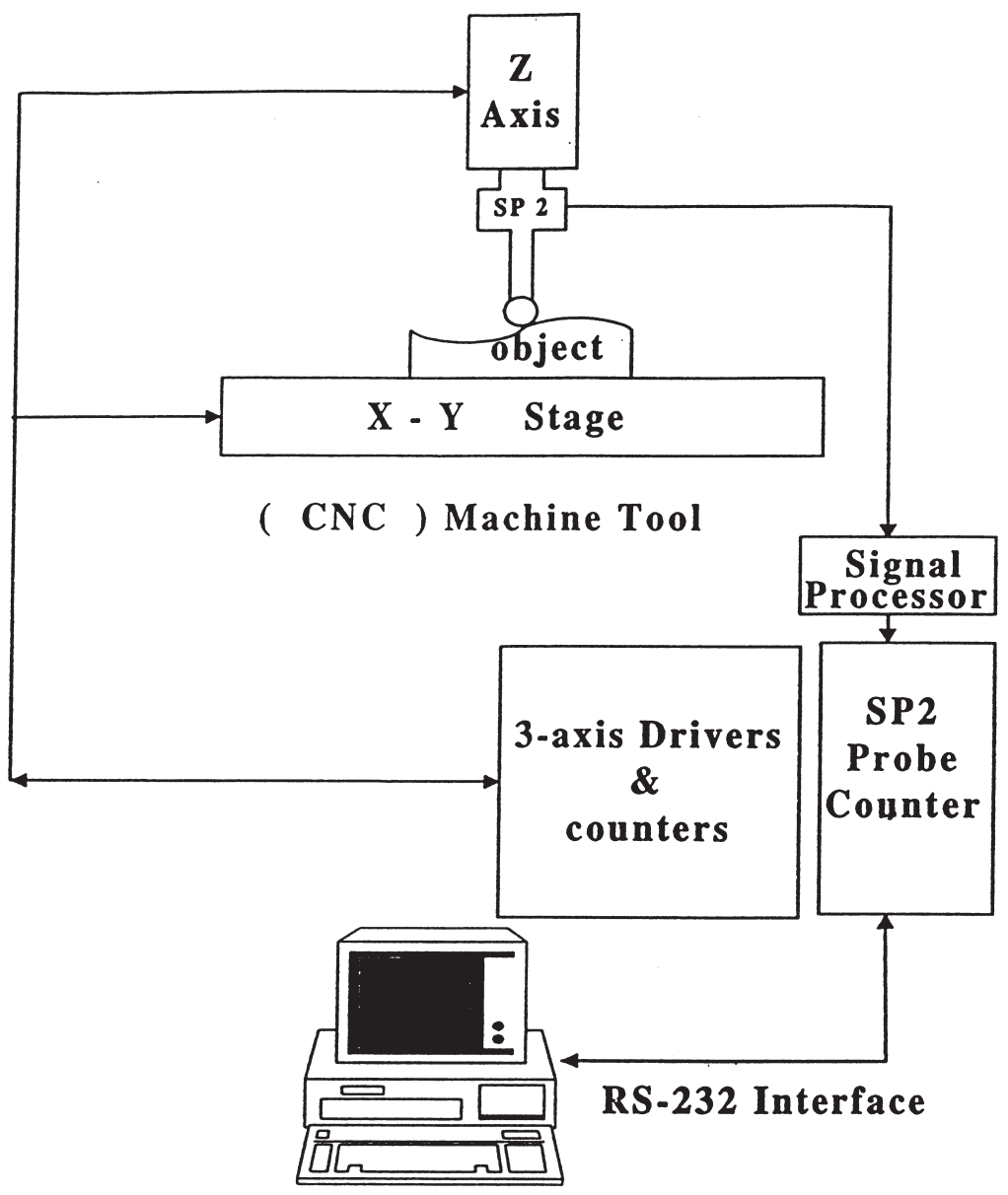

IBM PC / 486

Fig. 1. Schematic diagram for the measuring system.

with the probe's contact pressure. In our work, the sampling period was set $0.02 \mathrm{~s}$ so as to determine exactly when the probe becomes stable. The probe scans line by line from the selected coordinate origin to the last point, as shown in Fig. 2. However, it was found very difficult to maintain a continuous measuring path during the process of surface measurement with the conventional PID control. This difficulty was overcome when employing a fuzzy logic control scheme. The difference in the results between implementing these two control schemes will be described later.

\section{Employment of fuzzy logic control}

The concept of fuzzy sets was first introduced by Lotfi Zadeh [6] in 1965. In 1974, E.H. Mamdani originated a fuzzy control system for steam engines, using his inference procedure. 


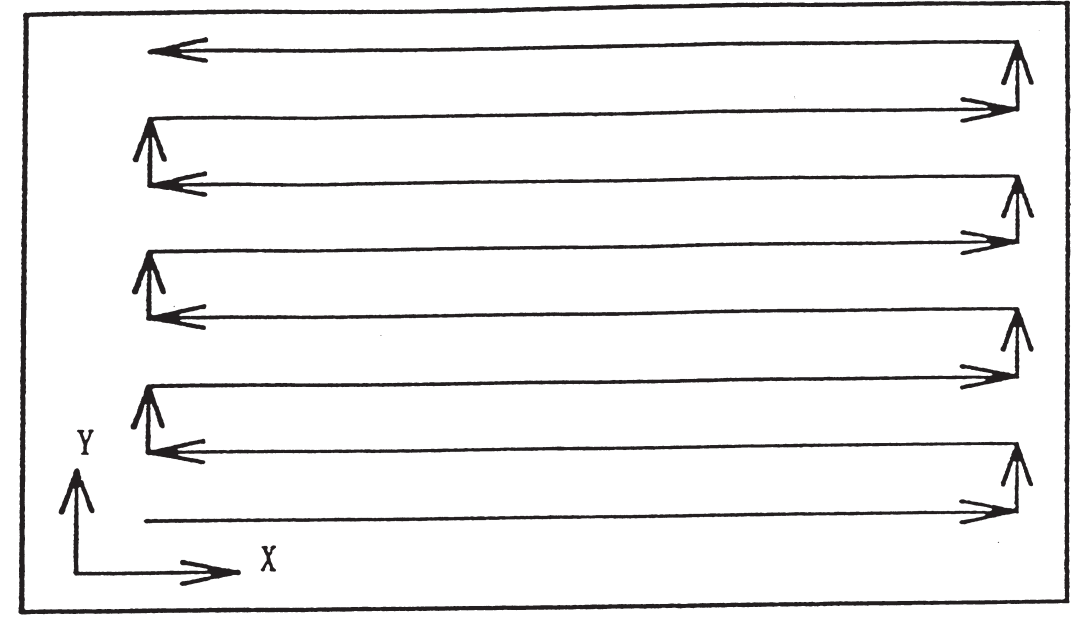

Fig. 2. The probe's scanning route.

Japan began developing fuzzy systems in the 1980s, following the Europe's pioneering work. Japanese developers focused almost all of their efforts on designing alternatives to conventional control logic. The result was a new fuzzy control scheme that provided nonlinear control action with very simple mechanism. The main advantage is that fuzzy control rules are more conveniently formulated in linguistic rather than numerical terms.

\subsection{Fundamentals of fuzzy logic control}

Fig. 3 shows a basic configuration of fuzzy logic controller which can be divided into four parts: fuzzification, knowledge base, decision making logic and defuzzification [7]. They are briefly described as follows.

1. Fuzzification takes the feedback data of state variables and maps the data to the fuzzy space which converts data to linguistic description. Fuzzification plays an important role in dealing with uncertain information which might be objective or subjective. The state variables here are the probe's $Z$ axis shift error, $E$ and the change of error, $\Delta E$. In this work, all these values are transformed from numerical data to five linguistic description groups as $N B$ (negative big), $N S$ (negative small), $Z O$ (zero), $P S$ (positive small) and $P B$ (positive big). These five groups are classified according to the predefined triangular-shaped membership functions, as shown in Fig. 4.

2. The knowledge base consists of data base and linguistic (fuzzy) control rule base. The former does discretization or normalization of universes of discourse, and makes a choice of the membership function of a primary fuzzy set. The latter uses control rules as fuzzy if-then rules which can be deduced from the observation of human controller's actions in terms of inputoutput operating data [8].

3. Decision making logic is the kernel of a fuzzy logic controller; it has the capability of simulating human decision making based on fuzzy concepts and of inferring fuzzy control actions employing fuzzy implication and the rules of inference in fuzzy logic. 


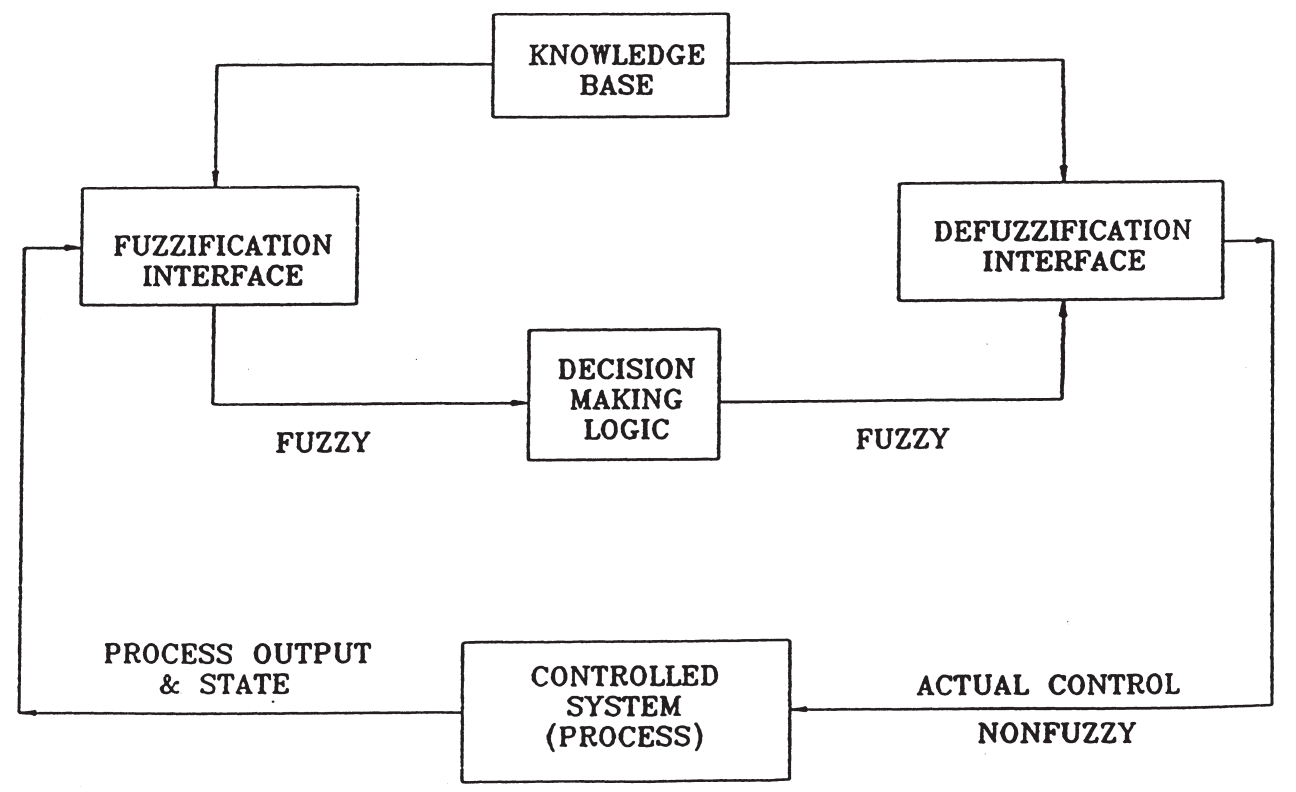

Fig. 3. Fuzzy logic controller's structure.

4. Defuzzification is a mapping from a space of fuzzy control actions defined over an output universe of discourse into a space of non-fuzzy (crisp) control actions. Therefore, the output of this process is the controlled values of the probe's Z-axis movement. Adopting the widely used Center of Area method, a single control value can be obtained from the following formula [7]:

$$
\begin{aligned}
& Z=[N B \times(-1.0)+N S \times(-0.5)+Z O \times(0)+P S \times(0.5)+P B \times(1.0)] / \\
& (N B+N S+Z O+P S+P B)
\end{aligned}
$$

where $N B, N S, Z O, P S$ and $P B$ are the degree of membership functions (negative big, negative small, zero, positive small, and positive big).

\subsection{Illustrative example}

Referring to Fig. 4, there are two input-variable membership functions, $E$ and $\Delta E$, and one output-variable membership function, $Z$, where $E$ is the probe's $z$-axis shift error, $\Delta E$ is the change of $E$, and $Z$ is the controlled movement in $Z$ direction. To illustrate how the fuzzy logic system works, let us assume that the first input variable $E=0.1(\mathrm{~mm})$ which results in two degree of membership function values, $Z O=0.8$, and $P S=0.2$, and the second input variable $\Delta E=0.3$ $(\mathrm{mm})$ which results in $Z O=0.4$, and $P S=0.6$, then a total of 25 fuzzy logic rules listed in Fig. 4 can be applied. In this case, there are four applicable rules.

Rule 1: If $E$ is $Z O$, and $\Delta E$ is also $Z O$, then the output variable $Z$ should be $Z O$, Rule 2: If $E$ is $Z O$, and $\Delta E$ is also $P S$, then the output variable $Z$ should be $P S$, 

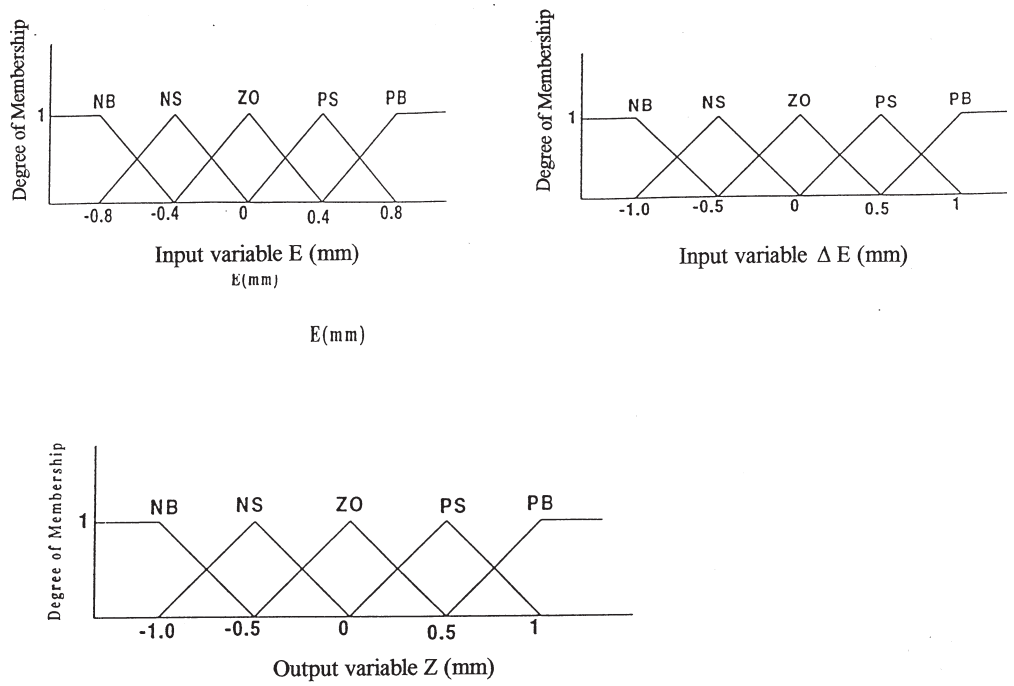

\begin{tabular}{|c|c|c|c|c|c|}
\hline 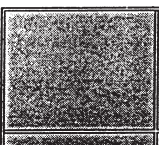 & \multicolumn{5}{|c|}{ 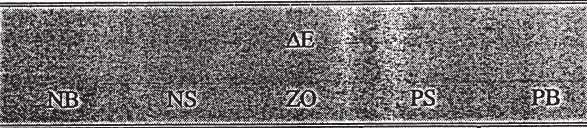 } \\
\hline $6 \mathrm{NB}$ & NB & NB & NB & NS & $\mathrm{ZO}$ \\
\hline NS & NB & NB & NS & $\mathrm{ZO}$ & PS \\
\hline $\mathrm{H}_{\mathrm{B}} \quad \mathrm{zO}$ & NB & NS & $\mathrm{ZO}$ & PS & PB \\
\hline C $\mathrm{PS}$ & NS & $\mathrm{ZO}$ & PS & PB & PB \\
\hline $6 \quad \mathrm{~PB}$ & PS & PB & PB & PB & PB \\
\hline
\end{tabular}

E: Positional Error; $\triangle \mathrm{E}$ : Change of Positional Error; PB: Positively Big;

PS: Positively Small; ZO: Zero; NB: Negatively Big; NS: Negatively Small

Fig. 4. Fuzzy membership functions and rule base.

Rule 3: If $E$ is $P S$, and $\Delta E$ is also $Z O$, then the output variable $Z$ should be $P S$, Rule 4: If $E$ is $P S$, and $\Delta E$ is also $P S$, then the output variable $Z$ should be $P B$.

Using Mamdani's minimum operation [7], the output variable $Z$ will have the following three degree of membership function values, $Z O=0.4, P S=0.2$, and $P B=0.2$, which is then substituted into Eq. (1) for defuzzification to generate a crisp value of $Z=0.3 \mathrm{~mm}$, the needed controlled movement in positive $Z$ direction.

It is worthwhile to note that the value of $Z$ movement comes directly from the fuzzy controller, but we also wish to control the $X$-axis movement. This can be accomplished by using the scanning increment value and the fuzzy controller's output $Z$ value. For instance, if the increment in $X$ direction is set at $1.0 \mathrm{~mm}$ and the controlled $Z$ value is $0.8 \mathrm{~mm}$ (i.e. raising the probe in $Z$ direction by $0.8 \mathrm{~mm}$ ), then the corresponding $X$-axis movement should be $\sqrt{1^{2}-0.8^{2}}=0.6 \mathrm{~mm}$. In this case, the $X$ movement is less than the preset increment $1.0 \mathrm{~mm}$ due to a rapid change in surface 
curvature. As a result, the number of measuring points will increase when scanning along the $X$ axis. On the other hand, if the change of surface curvature is small, then the $Z$-axis need not be raised as much as $0.8 \mathrm{~mm}$. Accordingly, the $X$ movement will be bigger, closer to $1.0 \mathrm{~mm}$, and resulting in less measuring points. This automatically adjusted scanning increment is illustrated in Fig. 5.

\section{Difference between implementing PID and fuzzy logic control schemes}

The measuring accuracy for implementing PID control and fuzzy control schemes will be discussed later in the section of experimental results. In this section, we discuss only the advantage and disadvantage of using each scheme.

\subsection{With PID control}

The major difficulty in using the conventional PID control scheme is to maintain a continuous measuring path. We had to develop a surface tracing algorithm to predict the next measuring position to which the CNC should move to. The measuring paths were classified into five categories:

1. general path,

2. descendant path,

3. ascendant path,

4. lateral ascendant path,

5. lateral descendant path.

For instance, the general path is when the value of the probe's $Z$-axis counter is between 0.5 $\mathrm{mm}$ and $1.5 \mathrm{~mm}$. Thus, when the value goes beyond this range, the $\mathrm{CNC}$ machine tool would have to be raised or lowered in order to maintain a continuous movement. Our study showed the

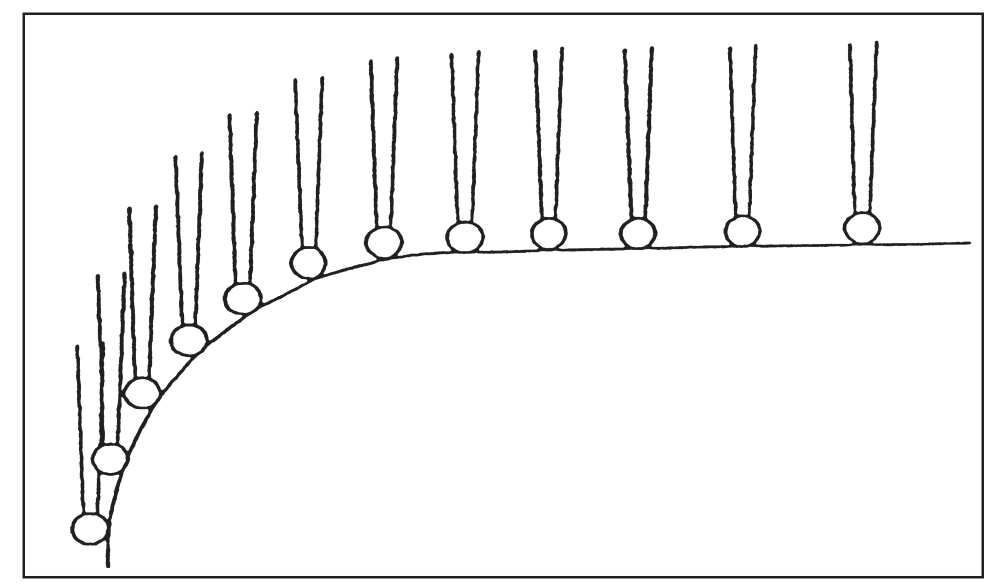

Fig. 5. Automatically adjusted scanning increment. 
ineffectiveness of PID control to keep the measuring process continuous. As a result, we had to develop hundreds of rules to try to prevent the CNC machine from stopping, and still failed to do so from time to time. The trouble occurred particularly when the probe was moving laterally on a steep surface. It was always necessary to create a new rule whenever the probe failed to move further.

\subsection{With fuzzy logic control}

The beauty of fuzzy logic control is that it is not required to specify the transfer function for the controlled plant in advance. This control scheme is very well suited for surface measurement in which the surface curvature can be very different from location to location.

The fuzzy logic control (FLC) rules are intuitive and easy to write. The controller can be designed with several trials on modifying the fuzzy membership functions and rule base. More importantly, the controller worked very well, and was able to keep the measuring process continuous. We also found that with the fuzzy logic control there was no need to classify the moving path into five categories. Furthermore, the employment of FLC makes it possible to automatically adjust the scanning increment, which in turns saves measuring time.

\section{Free form surface determination}

Due to unequal spacing between measuring points, the free form surface determination requires data preprocessing work, which is divided into three parts: (1) re-sampling, (2) probe center's coordinate transformation, and (3) free form surface reconstruction.

\subsection{Re-sampling (two-step process)}

The measured points usually form irregular grids or meshes as shown in Fig. 6(a). To be able to determine the surface normal everywhere on a surface under inspection, these points need to be re-sampled or reassembled through an interpolation scheme such as cubic splines or bilinear. The former is a parametric approach that guarantees the fitted surface to pass through all data points, while the latter is a much simpler and time saving process used in Cartesian coordinates.

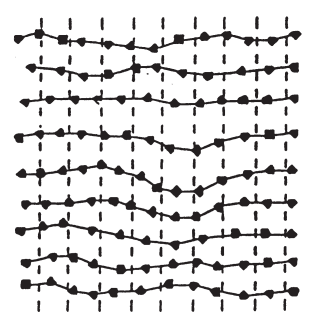

(a) Original Data Points

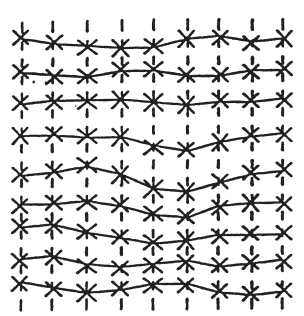

(b) New Meshes after Step 1

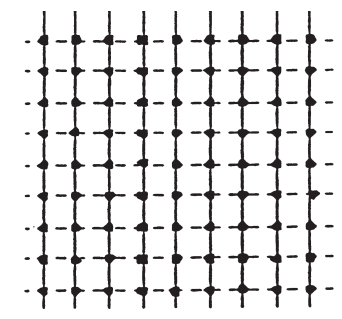

(c) Final Meshes after:Step 2

Fig. 6. Re-sampling process. 
The first step is to re-sample all the data with equal spacing in $X$ direction and interpolating the $Y$ and $Z$ data using the following equations;

$$
\begin{aligned}
& Y^{\prime}=Y_{i}+\left(Y_{i+1}-Y_{i}\right)\left(X-X_{i}\right) /\left(X_{i+1}-X_{i}\right) \\
& Z^{\prime}=Z_{i}+\left(Z_{i+1}-Z_{i}\right)\left(X-X_{i}\right) /\left(X_{i+1}-X_{i}\right)
\end{aligned}
$$

where $\left(X, Y^{\prime}, Z^{\prime}\right)$ is the new re-sampled point between two adjacent points $\left(X_{\mathrm{i}}, Y_{\mathrm{i}}, Z_{\mathrm{i}}\right)$ and $\left(X_{\mathrm{i}+}\right.$ ${ }_{1}, Y_{\mathrm{i}+1}, Z_{\mathrm{i}+1}$ ). Fig. 6(b) shows the end result of step 1. Likewise, the second step is to resample the already equally spaced $X$ data with equal spacing in $Y$ direction and interpolating the $Z$ data as follows.

$$
Z=Z_{j}{ }^{\prime}+\left(Z_{j+1}{ }^{\prime}-Z_{j}{ }^{\prime}\right)\left(Y-Y_{j}{ }^{\prime}\right) /\left(Y_{i+j}{ }^{\prime}-Y_{j}{ }^{\prime}\right)
$$

By doing so, $(X, Y, Z)$ becomes the new and final re-sampled point with equal spacing in $X$ and $Y$ directions, as shown in Fig. 6(c).

\subsection{Error analysis}

To analyze the degree of measuring error, let us assume that the probe size is carefully selected such that the probe radius is no larger than the smallest radius of curvature. Thus, the maximum measuring error should occur when the probe radius happens to be the same as the radius of curvature (see Fig. 7). When measuring a curve, the maximum error in determining the $Z$ value can be expressed by

$$
E_{\text {curve }}=R-\sqrt{R^{2}-(0.5 D)^{2}}
$$

where $R$ is the probe radius and $D$ is the sampling distance. Extending this expression to free form surface measurement, the maximum error due to the use of bilinear interpolation can be expressed by

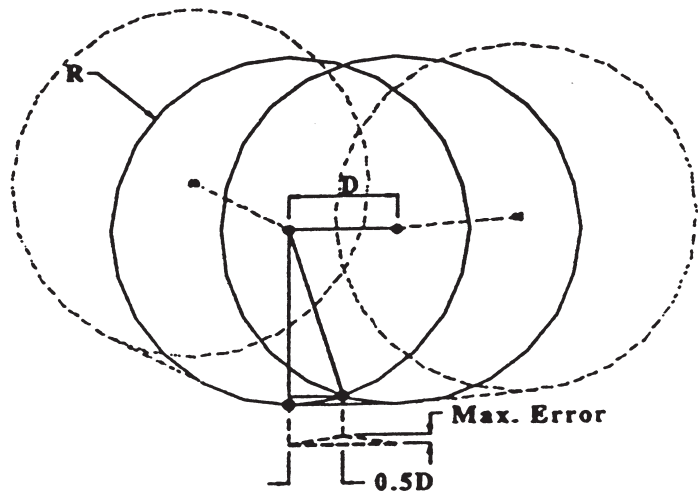

(s)

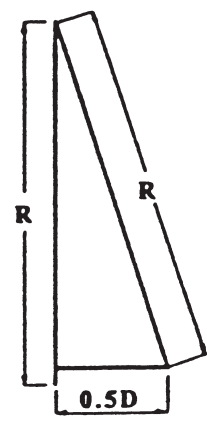

(b)

Fig. 7. Maximum error calculation. 


$$
E_{\text {surface }}=R-\sqrt{R^{2}-(D / \sqrt{2})^{2}}
$$

In our work, we usually used $R=2.5 \mathrm{~mm}$ and $D=0.5 \mathrm{~mm}$, which results in acceptable maximum error of $0.025 \mathrm{~mm}(25 \mu \mathrm{m})$ as calculated from Eq. (6). Here the sampling distance $D$ is always chosen to be smaller than or equal to the scanning increment (typically between 0.5 and $1.0 \mathrm{~mm}$ ).

\subsection{Probe center's coordinate transformation}

When using the scanning probe, the position data thus obtained is with respect to the probe center. Thus, the center's coordinate will have to be transformed to the coordinates containing the measured object's contact point every time (see Fig. 8). The perpendicular distance between the probe center and the contact point is the probe radius $R$ if the probe is normal to the surface at the contact point. It is desired to keep the probe normal to the scanned surface all the time. Therefore, the first task is to calculate the surface normal vector of any contact point. Let $(X, Y$, $Z$ ) and $(x, y, z)$ be the contact point's coordinates and the probe center's coordinates, respectively, and $N(i, j, k)$ be the surface normal vector. Then the coordinate transformation can be given by

$$
(X, Y, Z)=(x, y, z)-R N(i, j, k)
$$

Vector $N$ can be determined by taking the cross product of two surface tangent vectors (in $X$ and $Y$ directions) along the measured curved surface where the surface tangent can be obtained from differentiating the fitted surface with respect to $X$ and $Y$ axes. Nine re-sampled points in the neighborhood of an associated re-sampled point plus the point itself were fitted into the following general second-order surface equation

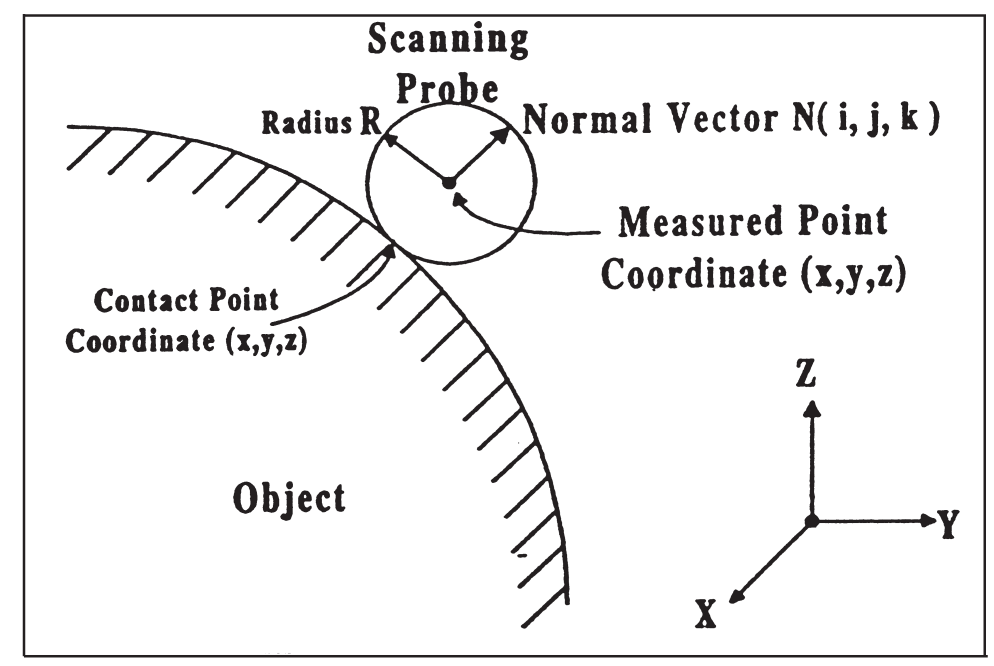

Fig. 8. Coordinate relationship between probe and object. 


$$
f(X, Y, Z)=a X^{2}+b Y^{2}+c Z^{2}+d X Y+e X Z+f Y Z+g X+h Y+k Z+t=0
$$

Then, the surface tangent vectors in $X$ and $Y$ directions, respectively are

$$
\begin{aligned}
& v_{x}=\partial f / \partial X=2 a X+d Y+e Z+g \\
& v_{y}=\partial f / \partial Y=2 b Y+d Y+f Z+h
\end{aligned}
$$

The associated surface normal vector can then be determined by taking the cross product of $v_{x}$ and $v_{y}$. It should be noted that the surface normal vector can be determined only with a fitted surface, and that the fitted surface is based on the re-sampled data points.

\subsection{Free form surface reconstruction}

In free form surface reconstruction, we use NURBS (Non-Uniform Rational B-Spline) [9], the most popular method in CAD/CAM systems. The rationale is that NURBS possess global and local flexibility and can represent complex surfaces. The term "rational" means that these functions are obtained by the "ratio" of two polynomials. They are invariant under projective transformations. A rational surface can be represented by the following expression[10]:

$$
p(s, t)=\frac{\sum_{i=0}^{n} \sum_{j=0}^{m} w_{i j} N_{i k}(s) N_{j l}(t) V_{i j}}{\sum_{i=0}^{n} \sum_{j=0}^{m} w_{i j} N_{i k}(s) N_{j l}(t)}
$$

where $N_{i k}(s)$ and $N_{j l}(t)$ represents the basic blending functions, $V_{i j}$, is the set of control points, and $w_{i j}$ is known as weights at each control point, which provides an additional degree of freedom for surface shaping.

NURBS are rational B-spline surfaces with a non-uniform knot vector. The general expression for NURBS given in Eq. (11) is for rational B-spline, except that the knot vector used is nonuniform. A non-uniform knot vector means that the knot vector is unequally spaced. A nonuniform knot vector sequence can be obtained by the introduction of multiple interior knot values,

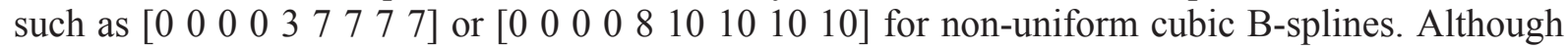
a uniform knot spacing is easy to use, there are disadvantages to the availability of non-uniform spacing for shape control in the design process. For example, undesirable oscillations may occur when using a uniform B-spine with highly unevenly spaced data.

The order of NURBS should be carefully selected. The higher the order is, the smoother the curve will be, but the interpolated data will be distorted (i.e. away from the original data points).

\section{Experimental results}

\subsection{Via a fuzzy logic controller}

To verify the system's measuring accuracy, we used an accurately machined part of known geometry. This part has two curved surfaces with constant radius of curvature, and two flat sur- 

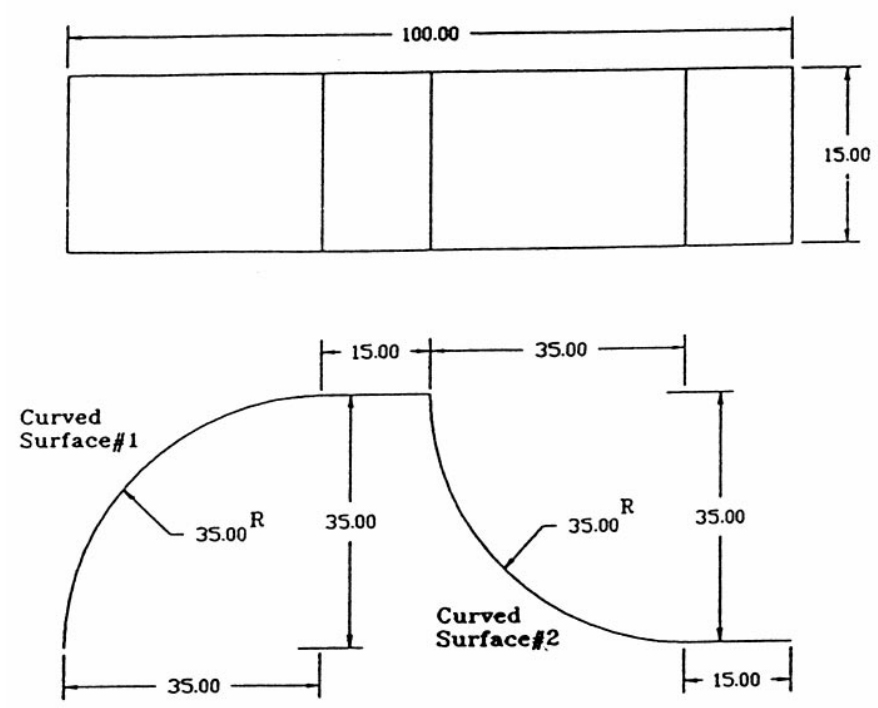

Fig. 9. Dimensions of the machined part for testing.

faces as shown in Fig. 9. In this experiment, we used a probe of $2.5 \mathrm{~mm}$ radius, and a maximum sampling distance of $0.5 \mathrm{~mm}$. Since this machined part is $15 \mathrm{~mm}$ thick, we equally divided the thickness into six groups. Each group represents a measuring path following curved surfaces \#1 (uphill) and then \#2 (downhill). Along each path, 40 measurements (i.e. samples) were taken. The points taken in surfaces \#1 and \#2 were fitted into an equation to find the actual radii, which were then compared with the nominal radius $(35 \mathrm{~mm})$. The measuring errors are listed in Tables $1-4$.

As shown above, the average measuring error for curves \#1 and \#2 is about $14 \mu \mathrm{m}$. Taking

Table 1

Measuring errors on curved surface \#1

\begin{tabular}{lll}
\hline Measuring path & Measured radius $(\mathrm{mm})$ & Measuring error $(\mathrm{mm})$ \\
\hline 1 (uphill) & 35.021 & 0.021 \\
2 (uphill) & 35.008 & 0.008 \\
3 (uphill) & 35.013 & 0.013 \\
4 (uphill) & 35.017 & 0.017 \\
5 (uphill) & 35.008 & 0.008 \\
6 (uphill) & 35.015 & 0.015 \\
1 (downhill) & 35.004 & 0.004 \\
2 (downhill) & 35.012 & 0.012 \\
3 (downhill) & 35.013 & 0.013 \\
4 (downhill) & 35.005 & 0.005 \\
5 (downhill) & 35.009 & 0.009 \\
6 (downhill) & 35.015 & 0.015 \\
\hline
\end{tabular}


Table 2

Summary of measuring errors on curved surface \#1

\begin{tabular}{lll}
\hline Measuring path & Average errors $(\mathrm{mm})$ & Maximum error $(\mathrm{mm})$ \\
\hline Uphill & 0.014 & 0.021 \\
Downhill & 0.010 & 0.015 \\
\hline
\end{tabular}

Table 3

Measuring errors on curved surface \#2

\begin{tabular}{lll}
\hline Measuring path & Measured radius $(\mathrm{mm})$ & Measuring error $(\mathrm{mm})$ \\
\hline 1 (uphill) & 35.017 & 0.017 \\
2 (uphill) & 35.012 & 0.012 \\
3 (uphill) & 35.008 & 0.008 \\
4 (uphill) & 35.021 & 0.021 \\
5 (uphill) & 35.012 & 0.012 \\
6 (uphill) & 35.009 & 0.009 \\
( downhill) & 35.017 & 0.017 \\
(downhill) & 35.019 & 0.019 \\
(downhill) & 35.022 & 0.022 \\
4 (downhill) & 35.013 & 0.013 \\
5 (downhill) & 35.018 & 0.018 \\
6 (downhill) & 35.021 & 0.021 \\
\hline
\end{tabular}

Table 4

Summary of measuring errors on curved surface \#2

\begin{tabular}{lll}
\hline Measuring path & Average error $(\mathrm{mm})$ & Maximum error $(\mathrm{mm})$ \\
\hline Uphill & 0.013 & 0.021 \\
Downhill & 0.018 & 0.022 \\
\hline
\end{tabular}

the maximum errors (average of about $20 \mu \mathrm{m}$ ) into account, we estimate that the presented fuzzylogic controlled measuring system can generally achieve the accuracy of 20-30 $\mu \mathrm{m}$.

In addition to the machined part of regular geometry, we also tested the capability of the measuring system on a free-form surface, a female model. The model is approximately $738 \mathrm{~mm}$ by $416 \mathrm{~mm}$. In this test, we used a probe of $6 \mathrm{~mm}$ radius, and a maximum sampling distance of $3 \mathrm{~mm}$. Fig. 10 shows the original 3-D plot, and the final 3-D plot after re-sampling and probe center coordinate transformation. The entire measuring process was very smooth with the fuzzy logic controller, and the reconstructed free form surface appears to be the same as the original. However, we did not quantify the measuring errors on this unknown geometry model. In order to quantify the errors, we would have to establish the referenced or master geometry by measuring the geometry point by point using a CMM. By doing so, the measuring errors from the CMM 


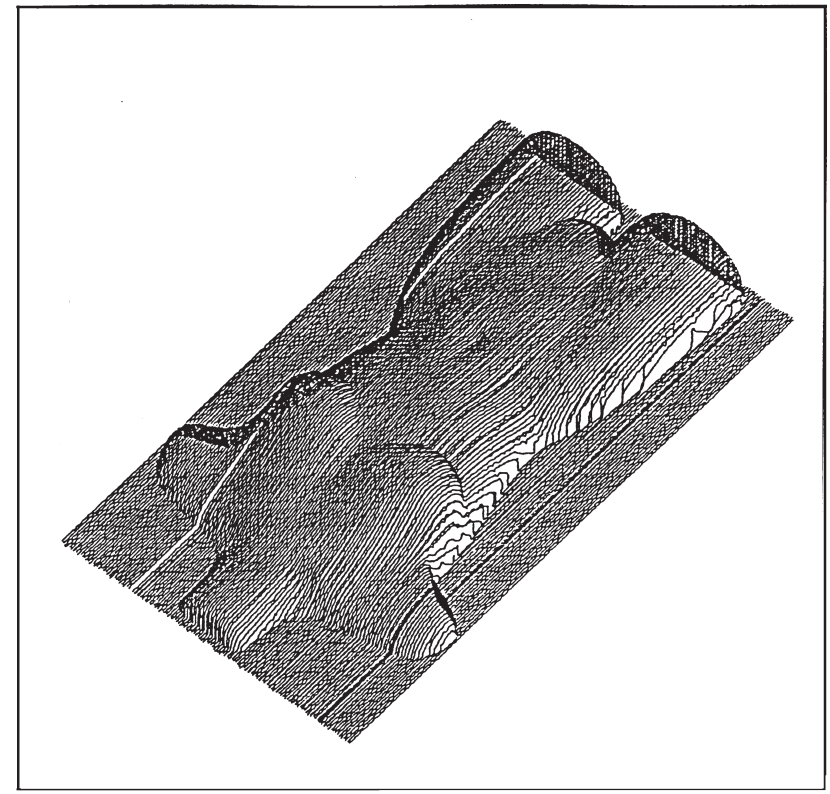

(a)

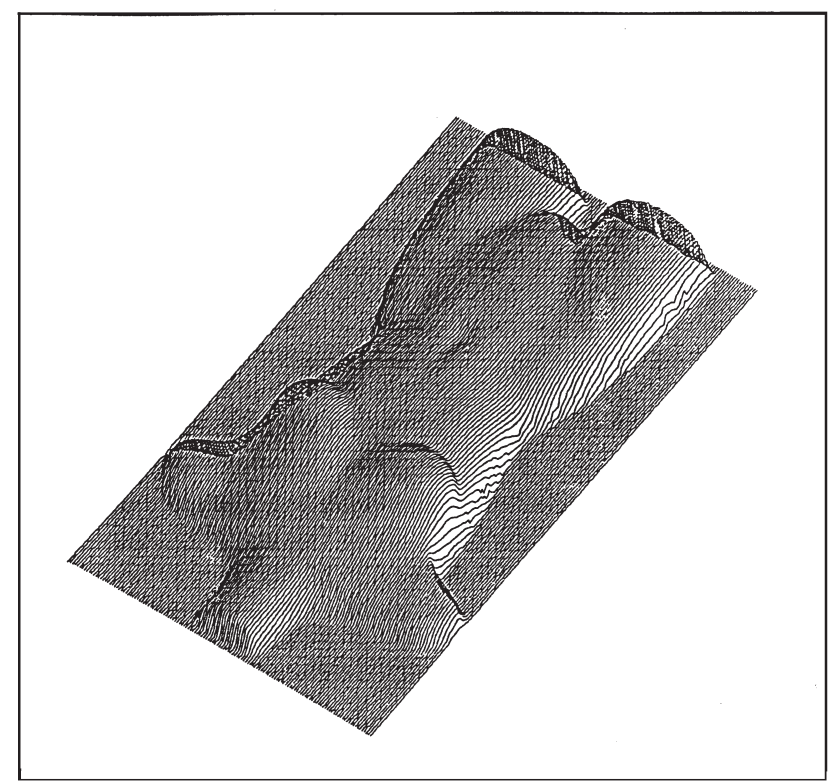

(b)

Fig. 10. The 3-D plot of a female model; (a) original; (b) measured by a scanning probe (re-sampling and coordinate transformation). 
itself will be introduced. Therefore, we believe that the best way to determine the measuring errors is to compare the measured data with known geometry such as an accurately machined part.

\subsection{Via a PID controller}

For the same machined part of regular geometry, some tests were conducted via a PID controller, and the errors are about the same as those obtained from using fuzzy logic controller. No substantial difference in measuring errors was expected between implementing these two control schemes. The only major difference is that the PID controller was often unable to keep the measuring process from being interrupted. It was very difficult to write good control rules for the probe to successfully scan laterally on a steep surface. We could not perform a thorough study on using a PID controller simply because it did not work properly with the scanning probe. The measuring accuracy may or may not be worse than a fuzzy logic controller, depending upon the degree of change in surface curvature. Our observation was that the measuring errors were about the same if the surface curvature did not change rapidly. On the other hand, if the curvature drastically changed, the probe was often stuck, which interrupted the measuring process. Therefore, it was difficult to compare the measuring accuracy between these two control schemes location by location.

\section{Conclusions}

This paper presented an on-line measuring system and methodology using a fuzzy-logic controlled scanning probe equipped on a CNC machine. As a result, the employment of fuzzy logic control gracefully minimizes the probe's unwanted lateral shift and successfully makes the entire measuring process continuous. One important and unique feature of the presented measuring system is the ability to automatically adjust the spacing of measurement points according to the degree of change in surface curvature. When the curvature changes substantially, the number of points increases, which improves the accuracy in surface measurement. On the other hand, when the curvature changes little, the number of points decreases, which saves the measuring time. All this cannot be accomplished by using the conventional PID control scheme. The employment of a fuzzy logic control scheme is mainly to improve the measuring stability and continuity.

With the scanning probe installed on a CNC machine, we have actually extended our work from measurement to reverse engineering, in that we converted the reconstructed 3-D surface data through standard graphics file formats, such as IGES (Initial Graphics Exchange Standard), to CAD/CAM software, such as AutoCAD and Smart CAM, for machining components. The only drawback of using a scanning probe is that a soft object might be damaged due to the preset contact pressure. The remedy to this drawback may be to use a force sensor with reaction force feedback control.

\section{Acknowledgements}

The first author acknowledges the support from the National Science Council in Taiwan under a grant number: NSC-86-2212-E-033-15. The second author would like to acknowledge the sup- 
port from Cleveland State University, USA under the EFFRD program, grant number 2107610660. Without their financial supports, this collaboration would not have been possible.

\section{References}

[1] J.A. Jalkio, Three dimensional inspection using multistripe structured light, Optical Engineering 24 (6) (1985) 966-974.

[2] M. Idesawa, T. Yatagai, T. Soma, Scanning moire method and automatic measurement of 3-D shapes, Applied Optics 16 (8) (1977) 2152-2162.

[3] M. Chang, D.S. Wan, On-line automated phase-measuring profilometry, Optics and Lasers in Engineering 15 (1991) 127-139.

[4] M. Chang, P.P. Lin, Noncontact sphericity measurement by means of phase shift interferometry, Transactions of ASME, Journal of Manufacturing Science and Engineering 18 (1996) 394-399.

[5] J.C. Lu, N.A. Duffie, J.G. Bollinger, Two dimensional tracing and measurement using touch trigger probes, Annals of the CIRP 31 (1982) 415.

[6] L.A. Zadeh, Fuzzy sets, Information and Control 8 (1965) 338-353.

[7] C.C. Lee, Fuzzy logic in control system: fuzzy logic controller-part I and II, IEEE Transactions, Systems, Man, and Cybernetics 20 (1990) 404-435.

[8] M. Sugeno, K. Murakami, An experimental study on fuzzy parking control using a model car, in: M. Sugeno (Ed.), Industrial Application of Fuzzy Control, North-Holland, Amsterdam, 1985, pp. 125-138.

[9] L. Piegl, W. Tiller, Curve and surface construction using rational B-splines, CAD 19 (9) (1987) 485-498.

[10] V.B. Anand, Computer Graphics and Geometric Modeling for Engineers, John Wiley, Chichester, 1993, pp. 313-314. 